\title{
X-Ray Emission Lines: Present and Future Experiments
}

\author{
H. Kunieda \\ Department of Astrophysics, Nagoya University, Furo-cho, Chikusa, \\ Nagoya, Japan
}

\begin{abstract}
After 34 years of X-ray astronomical observations, we approach the time when we will be able to explore AGN using line spectroscopy with newly developed technology and methods. In the beginning, X-rays from AGN were observed using proportional counters in a sort of photometric way, allowing us to determine that the continuum is a power law. This result suggested a predominance of non-thermal emission mechanisms in AGN. Rapid variability on time scales as short as 1000 seconds implied a small size for the X-ray emitting region, of order $10^{14} \mathrm{~cm}$, which is 3 orders of magnitude smaller than the optical emission-line regions.

The first detection of line emission from AGN was the $\mathrm{Fe}-\mathrm{K}$ line by the GINGA satellite. The line energy was $6.4 \mathrm{keV}$ and its equivalent width was about $150 \mathrm{eV}$ in Seyfert 1 galaxies. Detection became possible by use of large-area, low-noise proportional counters. The Fe- $\mathrm{K}$ line emission is important in X-ray astronomy because iron is rather abundant and this line is isolated in energy from neighboring lines.
\end{abstract}

The Fe-K line profile has been examined with the CCD detectors on board $A S C A$. Many Seyfert 1 galaxies exhibit a broad-line profile of more than $1 \mathrm{keV}$ width with an asymmetric tail on the low-energy side. This is explained as the fluorescence line from a relativistic accretion disk around a black hole, which is broadened by the Doppler motion and distorted by the strong gravitational field. The CCD detectors also revealed the absorption edges of warm material in the line of sight, which is ionized by the strong emission from AGN.

In the next decade, new spectrometers will be launched which can perform spectroscopy with $E / d E>100: A X A F$ in $1998, X M M$ in 1999 , and $A$ stro- $E$ in 2000. Dispersive spectrometers on board $A X A F$ and $X M M$ will be powerful tools for low-energy lines, while the calorimeter on board Astro- $E$ will examine the $\mathrm{Fe}-\mathrm{K}$ line profile. Spectral resolution $E / d E$ of several hundred will reveal the intensity ratio of satellite and resonance lines. This will give us physical parameters, such as the density and absolute size of surrounding matter. We hope that the structure of the nucleus will be more deeply understood using X-ray spectroscopy with new instruments, and we will come close to the level of optical spectroscopy, which has worked well in the study of the outskirts of AGN. 


\section{Introduction}

The first X-ray observation of an extra-solar object was performed with a rocket experiment by Rossi and Giacconi on 1962 June 12 (Giacconi et al. 1962). That was just 34 years ago, much shorter than the 4000-year history of China. In the first one-and-ahalf decades of X-ray astronomy, Galactic X-ray binaries and SNR were the major targets of observations, because $\mathrm{X}$-ray detection systems at that time were only sensitive enough for bright Galactic sources. As larger space vehicles were developed, X-ray detectors were improved in effective area, spatial resolution, spectral resolution, and energy range. In Fig. 1, previous $\mathrm{X}$-ray missions are summarized through the end of the 1980s.

The first intensive study of $\mathrm{X}$ rays from AGN was performed by HEAO-1 from 1977 through 1979 (Rothschild et al. 1979). Large proportional counters of about $2000 \mathrm{~cm}^{2}$ were on board and yielded X-ray continuum spectra over $2-30 \mathrm{keV}$ and variability data from AGN of mCrab or submCrab level. HEAO-1 detected hundreds of AGN above this level (Wood 1984). The time scale of $\mathrm{X}$-ray variability was found to be thousands or tens of thousands seconds for almost all AGN (Tennant \& Mushotzky 1983). This is much shorter than optical variability time scales and implies the $\mathrm{X}$-ray-emitting region is less than $10^{14} \mathrm{~cm}$ in size, based on light travel-time arguments.

Figure 2 is an example of the energy spectrum from Seyfert 1 galaxies (Mushotzky et al. 1980). It shows the incident photon spectrum after deconvolution from the observed counting-rate spectrum. The typical continuum spectrum can be described well by a power law with photon indices $\Gamma \approx 1.6-1.7$ 

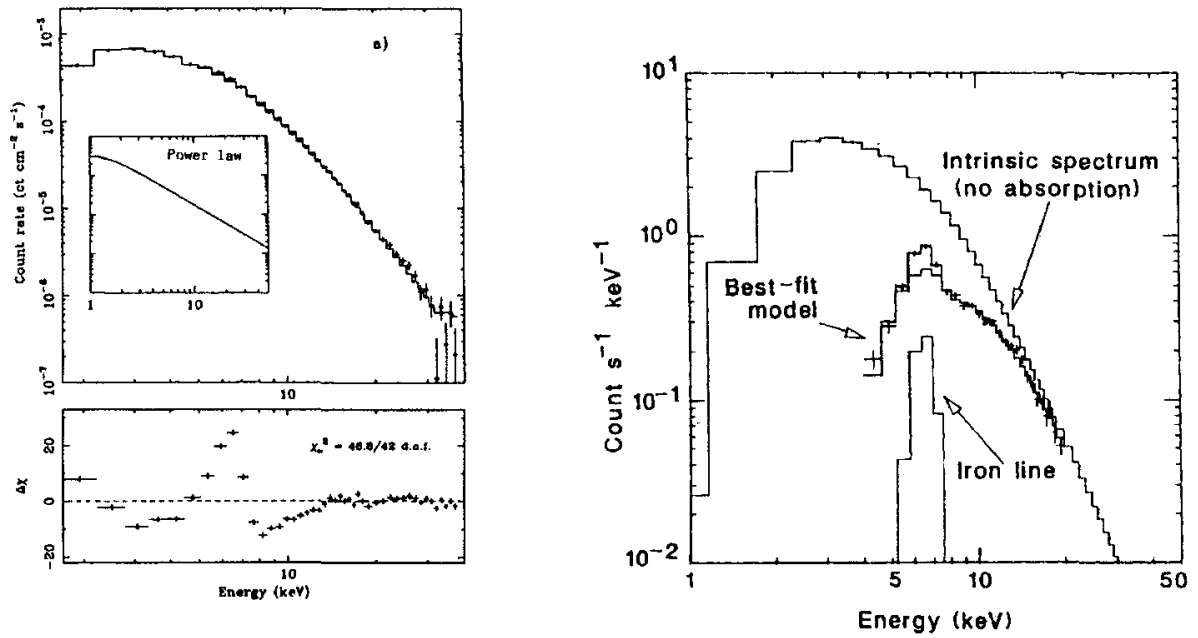

Figure 3. Composite of 27 Sy 1. Figure 4. Seyfert 2 spectrum (Mkn 3).

(Mushotzky et al. 1980). Such power-law spectra suggest a non-thermal emission mechanism for the central engine of AGN. On the other hand, the line sensitivity of proportional counters is so low that no line emission was detected from AGN. An exception is the oxygen trough in BL Lac objects (Canizares \& Kruper 1984).

The first satellite with an X-ray telescope, Einstein, was launched in 1978 (Giacconi et al. 1979). The effective area of the telescope (Fig. 6, below) was a few hundred $\mathrm{cm}^{2}$ below a few $\mathrm{keV}$, with spatial resolution of several arcseconds. We could see beautiful X-ray images of AGN. Einstein was equipped with a transmission grating, and crystal and solid-state spectrometers. Unfortunately, they did not yield much significant spectroscopic data from AGN mainly on account of small effective area.

The first detection of X-ray emission lines from many AGN was done by the Japan-UK GINGA satellite (1987-1991) (Makino et al. 1987, Turner et al. 1989). Though proportional counters of relatively poor energy resolution $(E / d E \approx 5$ at $6 \mathrm{keV}$ ) were the main detector system, its large effective area of $4000 \mathrm{~cm}^{2}$ and low background enabled us to detect iron $\mathrm{K}$ emission lines at 6-7 keV from many AGN. Initially, enhanced flux around $6-7 \mathrm{keV}$ and above $10 \mathrm{keV}$ was found in many individual Seyfert 1s. In order to get better statistics, Nandra \& Pounds (1994) accumulated X-ray spectra (Fig. 3) of 27 Seyfert 1 galaxies with GINGA. The lower panel presents the residual of the spectrum from a power-law model. There is an emission-line feature peaked at $6.4 \mathrm{keV}$ with an equivalent width of $127 \mathrm{eV}$. Since the K-line energy is $6.4 \mathrm{keV}$ for iron less ionized than Fe XVII, the observed line energy suggests it is a fluorescence line from cold material. 
The observed equivalent width is consistent with the illumination by the central source of optically thick material subtending about $2 \pi$ steradians (Awaki 1990).

Careful examination of the residual plot in Fig. 3 reveals that the iron line is located at the bottom of a dip in the spectrum. If the baseline is drawn at the bottom, an additional enhancement remains above $10 \mathrm{keV}$. This is explained by a reflection component from a cold wall illuminated by the continuum source at the nucleus.

Another important discovery from GINGA is the X-ray spectrum of Seyfert 2 galaxies. The continuum spectrum of $\mathrm{Mkn} 3$ is strongly attenuated at energies below several $\mathrm{keV}$ due to the absorption column of $10^{23.8}$ atoms $\mathrm{cm}^{-2}$ in the line of sight (Awaki et al. 1990), as shown in Fig. 4. This result supports the idea that the Seyfert 2 nucleus is obscured behind an absorbing torus. A relatively large equivalent width $(\sim 1 \mathrm{keV})$ iron $\mathrm{K}$-line can be attributed to the fluorescence from the inner wall of the absorption torus, which is illuminated by the central source.

\section{Current X-Ray Missions}

\subsection{Overview}

Current missions after 1990 are summarized in Fig. 5, along with major future missions. At this moment, four $\mathrm{X}$-ray satellites are operating: ROSAT, ASCA, $X T E$, and $S A X$. The latter two were launched within the past year, and will be discussed in the next section with other future missions. ROSAT, launched in 1990 , has an X-ray telescope which is sensitive to low-energy $\mathrm{X}$-rays below $2 \mathrm{keV}$ (Aschenbach 1987). The effective area is larger and spatial resolution is better than Einstein. Since the focal-plane detectors are the MCP and an imaging proportional counter (Pfeffermann et al. 1986), ROSAT gives fine images of AGN, but is not useful for AGN line spectroscopy. Therefore, I will concentrate on the $A S C A$ satellite and results from it in this section.

\subsection{Broad-Band X-Ray Telescope Missions}

In the $1980 \mathrm{~s}$, missions were equipped with large-area proportional counters of relatively poor energy resolution, or telescopes only sensitive at low energies. Einstein was sensitive below $4 \mathrm{keV}$, while $R O S A T$ is sensitive below $2 \mathrm{keV}$. The first X-ray mission with X-ray telescopes covering a broad energy band up to $10 \mathrm{keV}$, including the iron $\mathrm{K}$ lines, was the $B B X R T$ (Petre \& Serlemitsos 1985), launched on 1990 December 2 on the space shuttle Columbia as a part of a project named $A S T R O-1$, which also included UV telescopes. In Fig. 6, the effective area of the X-ray telescopes of $B B X R T$ and its successor $A S C A$ (Tanaka et al. 1994) are plotted against X-ray energies, in comparison to those of Einstein and ROSAT.

Since the incidence angles of X-ray telescopes have to be small to get adequate reflectivity, grazing optics are used at an angle of about $0^{\circ} .5$ for several-keV $\mathrm{X}$-rays. X-rays are reflected by the inner surface of cones and concentrated at a focus on the axis of the cones. To get a large effective area, hundreds of cones are nested co-axially. If the substrate is thick, a considerable fraction of the incident $\mathrm{X}$-rays is intercepted by them. For $B B X R T$, more than a hundred thin aluminum 


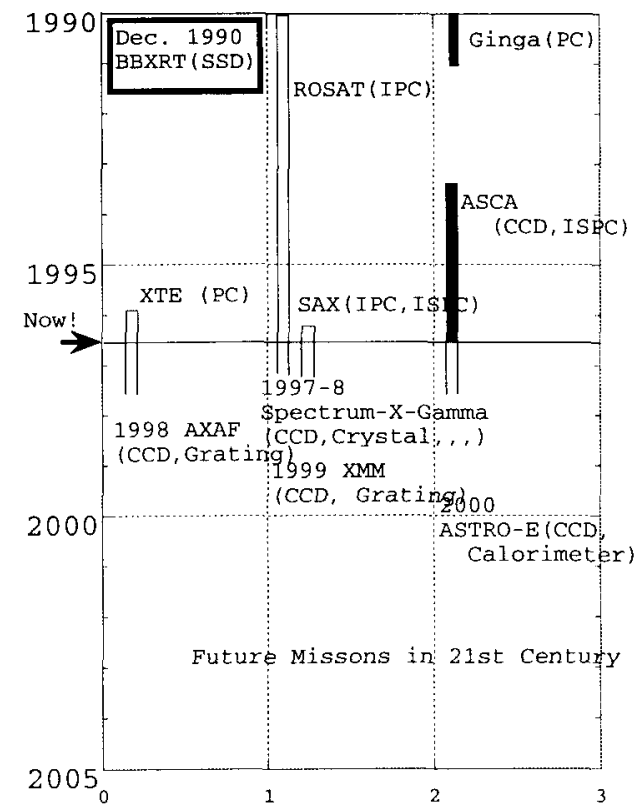

Figure 5. Current and future X-ray missions.

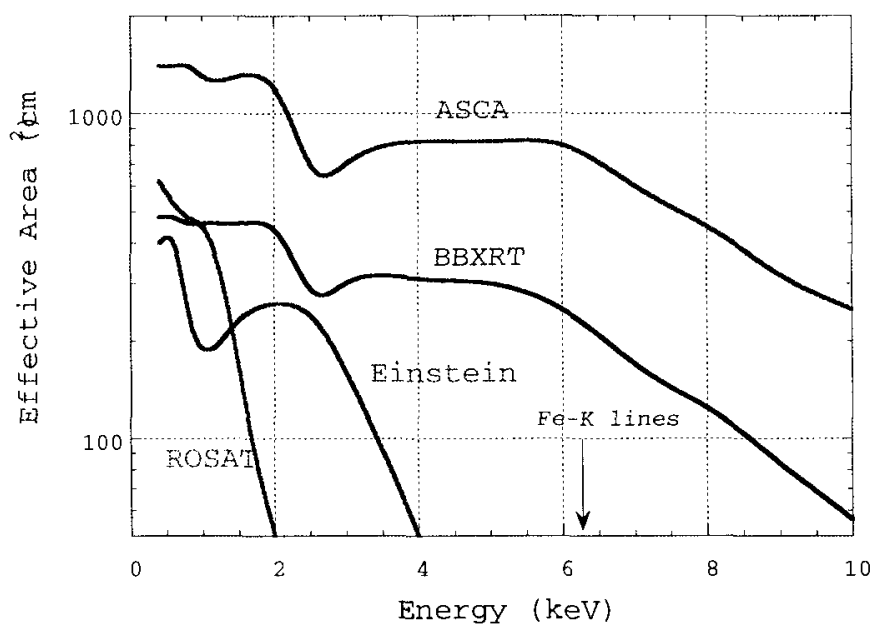

Figure 6. Effective area of previous X-ray telescopes. 


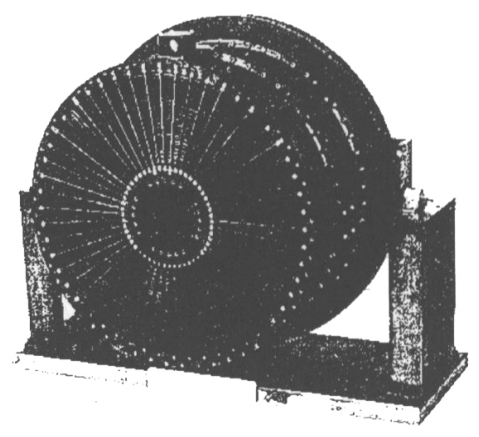

Figure 7. Nested thin foil mirror of $B B X R T$.

foils were installed with acrylic and Au coatings without polished surfaces. All the nested thin-foil mirror innovations are the work of Dr. Peter Serlemitsos at NASA/GSFC (Serlemitsos 1988). Figure 7 shows the X-ray telescope onboard $B B X R T$.

At the focal plane, $3.8 \mathrm{~m}$ away, there were segmented solid-state detectors, which provide ten times better energy resolution $(E / d E=50)$ than proportional counters. In the energy spectrum from the Seyfert 2 galaxy Mkn 3 (Fig. 4), a strong iron $\mathrm{K}$ line is seen at $6.4 \mathrm{keV}$ (Marshall et al. 1992). However, because of the short duration of the mission (just 9 days in orbit), it was difficult to obtain any definitive answers regarding line spectroscopy of AGN.

\subsection{ASCA Satellite}

Based on the telescope design of $B B X R T$, X-ray groups at NASA/GSFC and Nagoya University collaborated to develop telescopes for $A S C A$ (Serlemitsos et al. 1995). Four nested thin-foil mirrors are installed on the $A S C A$ satellite. The effective diameter is $345 \mathrm{~mm}$ and the weight of each telescope is $10 \mathrm{~kg}$, half the weight of $B B X R T$. These mirrors provide more than twice the effective area of $B B X R T$ at energies up to $10 \mathrm{keV}$ (see Fig. 6). In the focal plane, $3.5 \mathrm{~m}$ away, two kinds of detectors are mounted. Two CCDs are installed as the sub-systems named SIS 0 and 1, developed by MIT, Osaka University, and ISAS (Burke et al. 1991). Imaging gas-scintillation proportional counters named GIS 2 and 3 were developed by Tokyo University and ISAS (Ohashi et al. 1996). ASCA is the first satellite with $\mathrm{X}$-ray telescopes to cover up to $10 \mathrm{keV}$, including the iron K-lines. It improved the sensitivity by concentrating photons at a small spot in the focal-plane detector and reducing the solid angle of the sky corresponding to each imaging pixel. It allows us to detect faint sources, such as low-luminosity AGNs, Seyfert 2 galaxies, and distant quasars. The CCD is not only an imaging detector, but also a high energy-resolution spectrometer. The following results are all based on these technical improvements. 


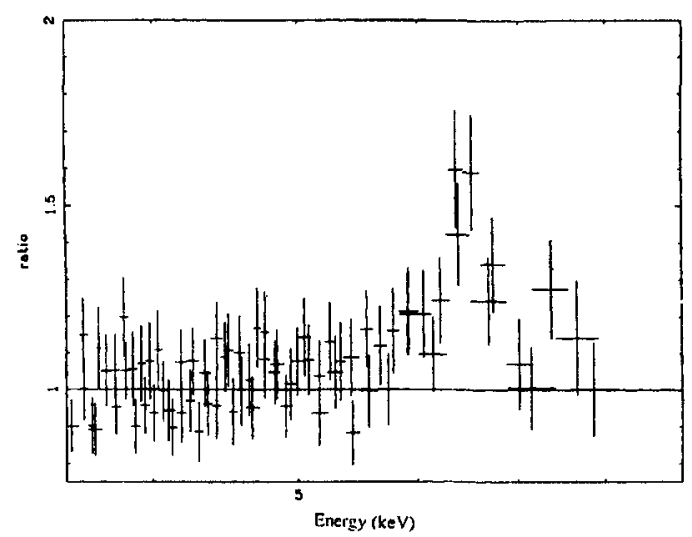

Figure 8. MGC-6-30-15. One-day integration with 1 SIS.

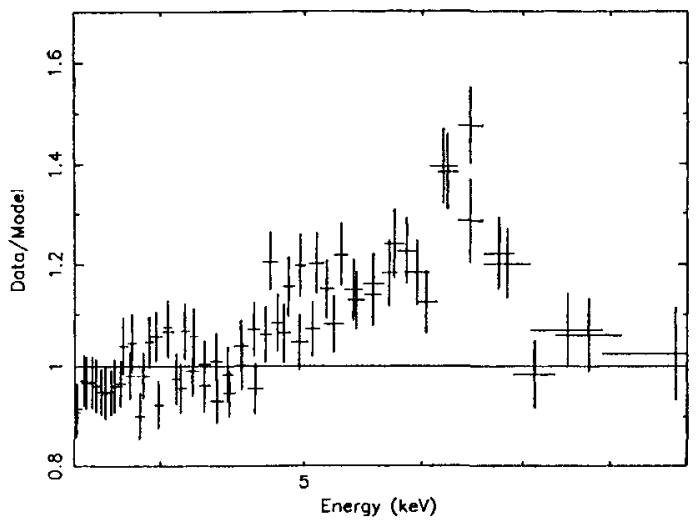

Figure 9. MGC-6-30-15. Two-day integration with 2 SIS.

\subsection{Broad Iron K-Line Feature}

At the beginning of $A S C A$ operations, we had planned spectroscopy of the iron $\mathrm{K}$ line discovered by GINGA. When we first looked at spectra of some Sy 1 galaxies, we were disappointed because the lines were not as clear and sharp as we expected with SIS, which has 10 times $(d E=120 \mathrm{eV})$ better energy resolution than proportional counters. If we apply a narrow-line model, an equivalent width of $80 \mathrm{eV}$ was obtained. Thus, if the lines are intrinsically narrow, half of the equivalent width observed with GINGA is missing in the $A S C A$ spectra of Seyfert 1s. At first, we thought this was due to poor counting statistics,

Figure 8 shows the ratio of the observational data to a power-law model for MCG-6 -30-15 for a one-day observation with one SIS detector (Fabian et al. 1994). The residuals are peaked at around $6.4 \mathrm{keV}$ with some broadening, although large error bars smear other features. Figure 9 shows the energy spectrum accumulated for two days with two SIS detectors (Kunieda 1995). Now a narrow peak and broad feature down to $5 \mathrm{keV}$ becomes clear. We decided to monitor the same source, MCG-6-30-15, for 5 days. Finally all the data are 


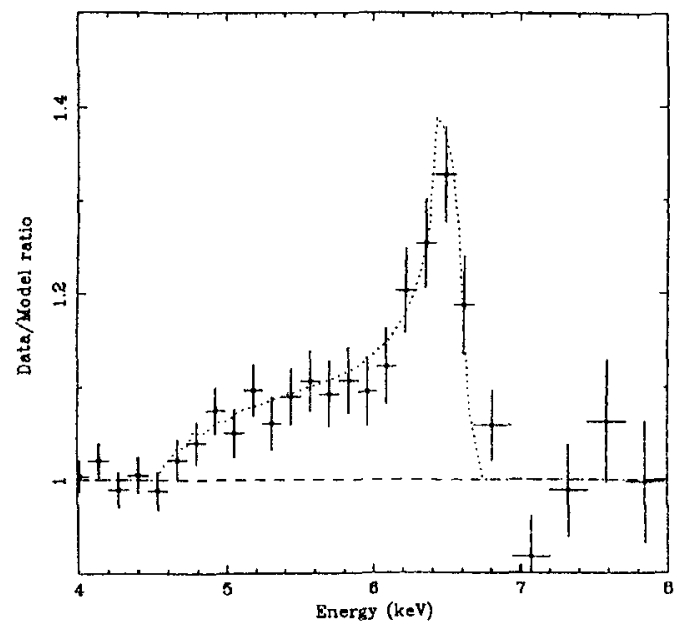

Figure 10. MGC-6-30-15. Five-day integration with 2 SIS.

accumulated to get the ratio plot shown in Fig. 10, where the narrow peak is found, together with broad component extending below $5 \mathrm{keV}$ (Tanaka et al. 1995).

The evolution of spectra from Figs. 8-10 represents the importance of good statistics for examination of fine features. Generally speaking, even with better energy resolution or spatial resolution, we cannot utilize the high performance if the effective area is small.

This feature is well described by line emission from an accretion disk around a massive black hole. The effect of strong gravity produces the long redward tail that extends down to $5 \mathrm{keV}$. Rapid rotation around the central object results in two peaks because of the orbital Doppler effect (this is inclination-dependent). Figure 11 shows the model calculation for the broad-line feature from a relativistic accretion disk around a black hole (Fabian et al. 1989). In the case of MCG-6-30-15, shown in Fig. 10, the accretion disk extends over the range 3-10 $R_{s}$, where $R_{s}=2 G M / c^{2}$, the Schwarzschild radius), and the inclination angle is about $30^{\circ}$. Halpern (1995) commented in the same issue of Nature that this is the long-awaited direct evidence of a strong gravitational field of a black hole.

More details of observations are presented by Nandra (this volume). He has compiled results from many Seyfert 1 galaxies and found the broad iron-line feature is common to all of them, with some variety of parameters (Nandra et al. 1996). Also, Iwasawa et al. (1996) report on the variations of the line profile in MCG-6-30-15, which are related to changes in the continuum luminosity.

\subsection{Warm Absorber}

Another important result from AGN with $A S C A$ is the detection of the absorption edges of ionized gas, the presence of which had been previously discovered using ROSAT (Nandra \& Pounds 1992). Figure 12 is the spectrum of MCG6-30-15 (Fabian et al. 1994) observed with $A S C A$ from one day of a two-day 


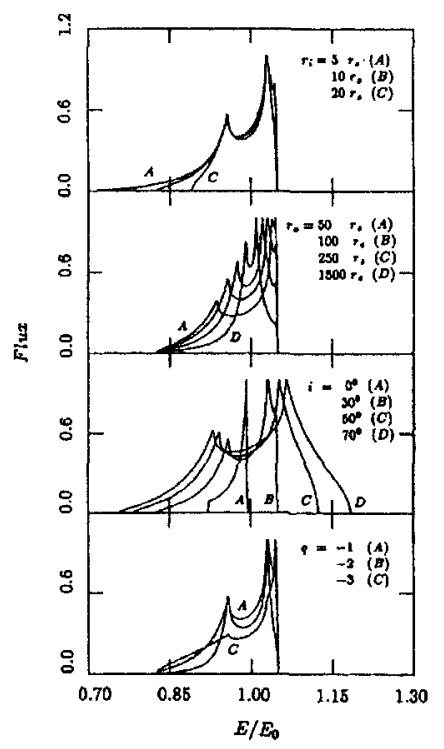

Figure 11. Model profile of the disk line.

observation with three-weeks separation. If we extend the power-law model determined above $3 \mathrm{keV}$, a clear deficit is seen in the $0.7-2 \mathrm{keV}$ range, and the spectrum is a little shallower on the other day.

The residual from the power law is plotted for both days in the lower panel. There are two steps at $0.74 \mathrm{keV}$ and $0.87 \mathrm{keV}$, which correspond to the O VII and $\mathrm{O}$ VIII edges, respectively. These spectral features can be modeled by gas in the line of sight which has been partly photoionized by the strong AGN continuum emission. If we apply such a model, an ionization parameter $\Xi \approx 40$ is obtained. It is clear that the ionization state has changed between the two observations, which were separated by 23 days. More detailed results from the 5-day observation of MCG-6-30-15 are presented by Ohtani et al. (1996).

These results mark the first time that the energy resolution of the $A S C A$ SIS was used to reveal edge energies and depth, indicating how much $O$ VII and $\mathrm{O}$ VIII are intervening in the line of sight.

\subsection{Emission Lines from Photoionized/Thermal Plasmas}

In this section, two more examples of emission lines from AGN are presented. The ASCA spectra from the Seyfert 2 galaxy Mkn 3 seem to have two components (Iwasawa et al. 1994). One is a strongly absorbed hard spectrum and the other is a soft component with emission lines. Both continuum components can be explained by the same power-law index. The former is assumed to be the direct component from the central source behind the absorber, and the latter is the component scattered by electrons. Since these lines are attributed to highly ionized ions, and they likely originate in material photoionized by the central source. 


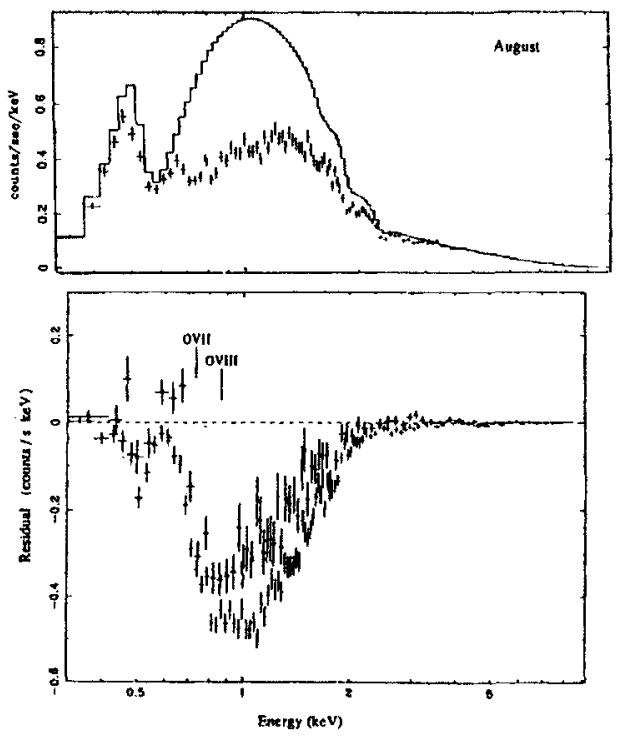

Figure 12. Warm absorber in MCG-6-30-15.

Emission lines from hot plasmas in starburst galaxies provide a final example. M 82 exhibits a soft thermal component and emission lines together with an absorbed hard component (Tsuru et al. 1994). The line ratio of different ionization states is consistent with a thermal continuum in general. These hot plasmas are not produced by the central engine, but created by the starburst activity. Thus, X-ray observations are important to determine the origin of plasmas surrounding the nucleus.

\section{Future Missions}

\subsection{Overview}

Future missions are summarized in Fig. 5. $X T E$ and $S A X$ were launched within the last year, and have just started their normal observation modes, so few new results from AGN have been reported so far. I will briefly discuss these two missions, together with Spectrum X-Gamma, whose launch is scheduled in 1997-98. There are three more missions being prepared for launch in this century: $A X A F$ in $1998, X M M$ in 1999 , and Astro- $E$ in 2000. I will concentrate on these missions because of their high performance in X-ray line spectroscopy. Some simulated Astro-E spectra will be shown to demonstrate the expected results for AGN. I will then conclude this paper with a discussion of some of the developments in X-ray technology for the missions in the 21st century.

\subsection{Rossi-XTE, SAX, and Spectrum-X-Gamma}

$X T E$ was launched on 1995 December 30 , and has been renamed the Rossi $X$-Ray Timing Explorer (RXTE). It is equipped with large-area proportional counters 
(PCA) of area $6250 \mathrm{~cm}^{2}$, scintillation counters (HEXTE) of area $1600 \mathrm{~cm}^{2}$, and an all-sky monitor (ASM) of area $90 \mathrm{~cm}^{2}$. The detectors are designed for measuring time variability and for monitoring X-ray novae. The movable solar panels allow observation of almost all of the sky all the time. The PCA has a much larger area and a broader energy range (up to $60 \mathrm{keV}$ ) than GINGA, which is important for observation of AGN continuum spectra. XTE observations at high energies may reveal the hidden AGN activity based on the absorbed spectral shape and time variability. Coordinated observations of AGN with XTE and $A S C A$ are important to get broad-band energy spectra of AGN and to understand the emission mechanism. Up-to-date information on XTE can be found on the WWW Homepage http://heasarc.gsfc.nasa.gov/doc/xte/.

$S A X$ has soft $\mathrm{X}$-ray mirrors, with gas-scintillation proportional counters at the focus and scintillation detectors to cover the energy band $0.1-300 \mathrm{keV}$. They will be powerful not in the line spectroscopy, but rather in observing X-ray sources over an unprecedented broad energy range. Additional information can be found on the WWW Homepage http://www.sdc.asi.it/sax_main.html.

Spectrum-X-Gamma is a Danish-Russian-US collaborative mission. It consists of two large-area, high-throughput telescopes similar to the BBXRT/ASCAtype telescopes, but twice as large in all scales. There are many kinds of focalplane detectors, including proportional counters, a solid-state detector, and a polarimeter. The solid-state spectrometer will be useful for line spectroscopy of AGN, providing better statistics than $A S C A$. There are several more detector systems, such as JET-X, MOXE, EUVITA, and MART. Since it will be launched by a Russian rocket, it is not clear yet whether the launch will occur in 1997 or 1998.

\subsection{AXAF}

The next giant $\mathrm{X}$-ray mission for the US is the long-awaited $A X A F$. The current design, after many changes forced by budget cuts, is shown in the WWW home page (see Table 1, below). The expected performance of $A X A F$ is is summarized in Table 1.

The main purpose of $A X A F$ is the high-resolution $\left(0^{\prime \prime} .5\right)$ imaging, also at high energies but with limited effective area; this instrument was originally called $A X A F-I$, which was to be a complement to a spectroscopy-oriented companion called $A X A F-S$. The polished X-ray telescope has a maximum diameter of $1.2 \mathrm{~m}$ and focal length of about $10 \mathrm{~m}$. The effective area is $1000 \mathrm{~cm}^{2}$ at $1.5 \mathrm{keV}$ and 250 $\mathrm{cm}^{2}$ at $7 \mathrm{keV}$ (see Fig. 18, below). For spectroscopy, three types of transmission gratings (LEG, MEG, HEG) are prepared which will give resolving power $E / d E$ more than several hundred (Fig. 20, below), while their total effective area is less than $100 \mathrm{~cm}^{2}$ (see Fig. 18).

Details of high-resolution X-ray spectroscopy of $A X A F$ are discussed by Fabbiano (this volume). Some numbers are also shown in Table 1 for comparison with $X M M$ and Astro-E.

\subsection{XMM}

The next giant $\mathrm{X}$-ray mission for ESA is $X M M$. They aim to achieve a large effective area with medium spatial resolution (a few tens of arcseconds). Three identical telescopes are made of 58 replica mirror shells. The diameter is $70 \mathrm{~cm}$ 

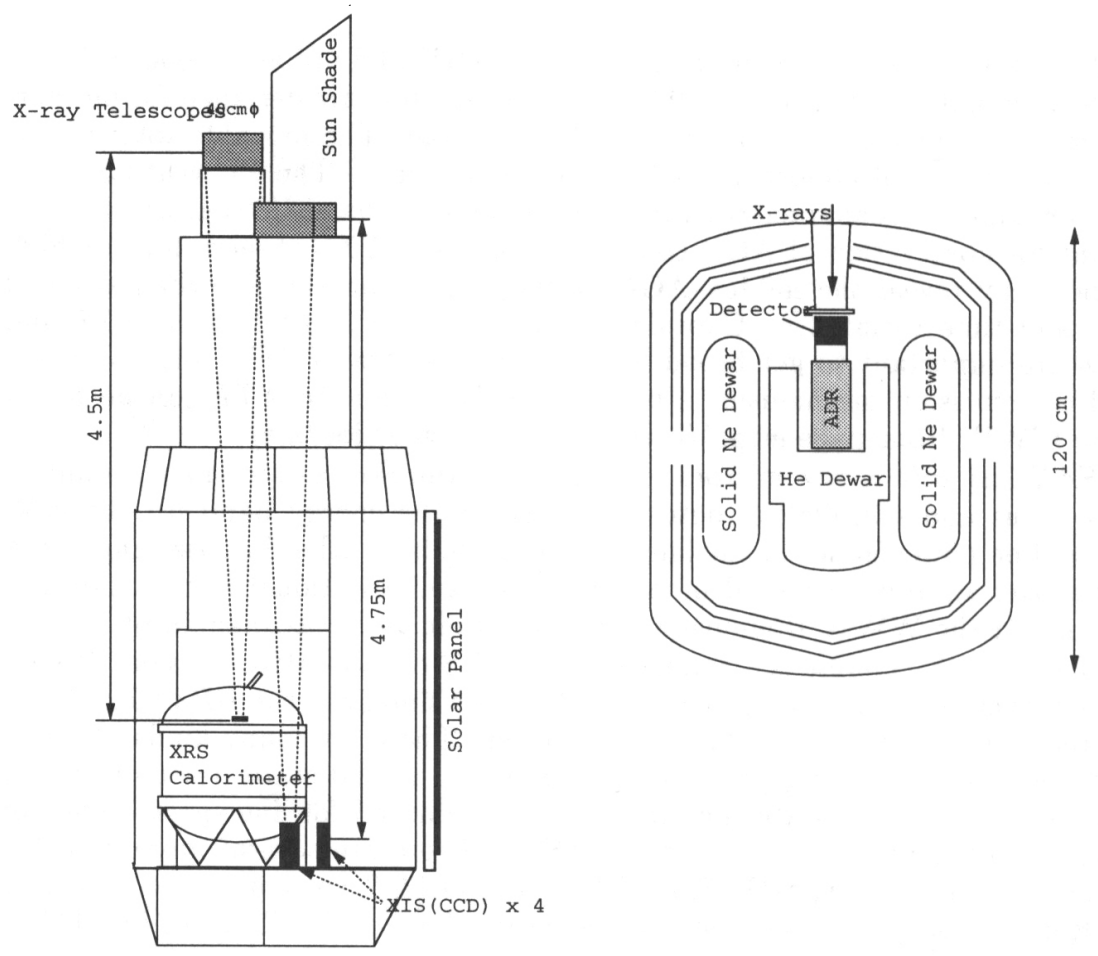

Figure 13. Structure of Astro-E satellite. Figure 14. Astro-E XRS system.

and the focal length is $7.5 \mathrm{~m}$. The effective area is $5000 \mathrm{~cm}^{2}$ at $1.5 \mathrm{keV}$ and $2500 \mathrm{~cm}^{2}$ at $7 \mathrm{keV}$ (see Fig. 18). For spectroscopy, the reflective grating spectrometer (RGS) is prepared for one of three telescopes to give resolving power $E / d E>300$ for X-rays below $2 \mathrm{keV}$ (Fig. 20).

The other two telescopes have CCD cameras in the focal plane, which will provide the largest effective area and thus will be the best instrument to investigate the broad iron-line profile in AGN. The imaging spectroscopy of AGN started with ASCA SIS will be continued, but with better statistics and better spatial resolution than with $A S C A$.

\subsection{Astro-E}

I will discuss Astro-E in detail, because of my deep involvement in it, and thus simulation programs are available to me. Astro- $E$ has two kinds of mirror systems, XRS and XIS. The former consists of a telescope $40 \mathrm{~cm}$ in diameter, with a $4.5-\mathrm{m}$ focal length and a micro-calorimeter in the focal plane. The latter consists of four identical $40-\mathrm{cm}$ telescopes of $4.75-\mathrm{m}$ focal length with CCDs at the foci. The effective area is $550 \mathrm{~cm}^{2}$ at $1.5 \mathrm{keV}$ and $400 \mathrm{~cm}^{2}$ at $7 \mathrm{keV}$ for XRS, and $2200 \mathrm{~cm}^{2}$ and $1400 \mathrm{~cm}^{2}$ for four sets of XIS (see Fig. 18).

The micro-calorimeter developed by NASA/GSFC provides resolving power of 500 at $6 \mathrm{keV}$ at the operating temperature of $60 \mathrm{mK}$. Therefore, it has to be 

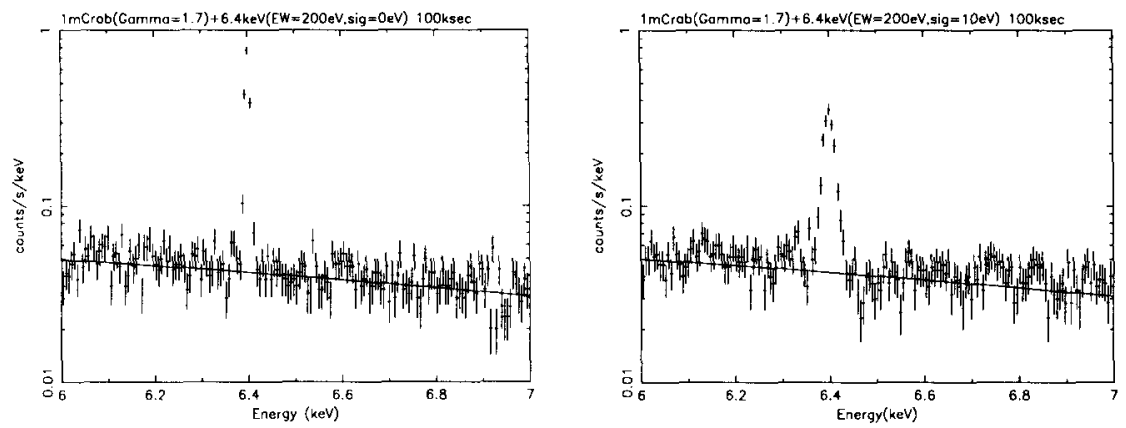

Figure 15. Left: Zero-width line by XRS. Right: $10 \mathrm{eV}$ width line.

cooled by an adiabatic de-magnetization refrigerator (ADR), a He dewar, and a solid Ne dewar (Fig. 14). Since it is a non-dispersive spectrometer, its resolving power becomes better at high energies (Fig. 20), and it is also capable of highresolution spectroscopy of extended sources. The detector array will allow us also to perform high-resolution imaging spectroscopy. The four XIS will provide more than four times as large an effective area as two sets of $A S C A$ SIS, with new CCDs with thicker depletion layers.

\subsection{Simulations}

Following are simulated results of the calorimeter onboard Astro- $E$ to demonstrate the performance of future missions with a resolving power $E / d E \geq 100$, which will be possible using $A X A F, X M M$, and $A S C A$. This simulation is supported by Dr. K. Arnaud at NASA/GSFC.

We assume a $1 \mathrm{mCrab}$ source with continuum power-law component having a photon index of $\Gamma=1.7$. Line emission is assumed to be at $6.4 \mathrm{keV}$ with an equivalent width of $200 \mathrm{eV}$. Figure 15 a shows the spectrum in the range $6-7 \mathrm{keV}$ (one data bin corresponds to $6 \mathrm{eV}$ ) which will be observed with the calorimeter (accumulation time of $100 \mathrm{ksec}$ ), when the intrinsic line width is zero. In other words, the width seen in Fig. 15a represents the energy resolution of XRS. Figure $15 \mathrm{~b}$ shows the case where the intrinsic width is assumed to be $10 \mathrm{eV}$. If you compare this to Fig. 15a, it is clear that XRS can recognize intrinsic broadening of $10 \mathrm{eV}$ or less. If $10 \mathrm{eV}$ intrinsic broadening is observed and can be attributed to the Doppler motion, the velocity corresponds to $470 \mathrm{~km} \mathrm{~s}^{-1}$.

The second example is the iron-line emission from the thermal plasma observed in the solar corona by the Hinotori satellite. The crystal spectrometer onboard Hinotori gave the high-resolution $(E / d E=12000)$ line profile shown in Fig. 16a (Tanaka 1986). If we input such a line profile into the XRS, we can expect the profile seen in Fig. 16b. Note that the abscissa is energy (in $\mathrm{keV}$ ) in this plot, while it was in wavelength in the previous plot. The largest peak at the shortest wavelength or highest energy is the resonance line. The lowest 

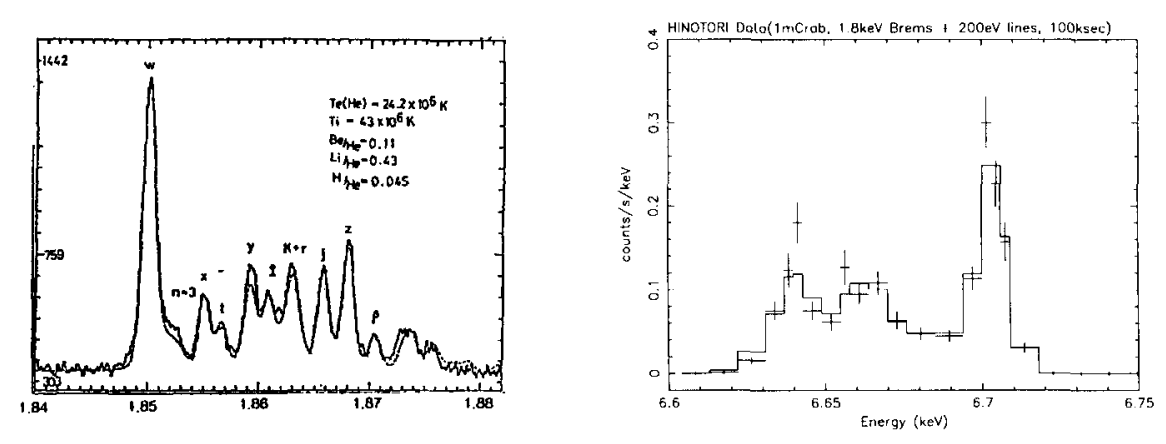

Figure 16. Left: Solar corona (Hinotori). Right: Coronal spectrum by XRS.

energy line is the forbidden line just at the side of the di-electric recombination line. Since the separation is comparable to the XRS resolution, both combine to make a large peak at the low-energy end in Fig. 16b. The intercombination lines in the middle are about $30 \mathrm{eV}$ away from both ends, and so they can be well separated by the XRS.

The third example is the broad iron $\mathrm{K}$-line profile discovered by $A S C A$. If we look at an AGN at an inclination of $30^{\circ}$, the high-energy end is so clear that the intrinsic line energy can be determined (Fig. 17a). However, search for the best parameters requires good statistics as well as good energy resolution. Among the three major missions in the next decade, I feel that the CCD system of $X M M$ will give the best-quality spectra to examine the disk structure, geometry, ionization state, and so on.

The edges of warm absorbers can be more easily recognized with better energy resolution, but not much impact on our understanding is expected. The emission lines from $\mathrm{O}$ VII and $\mathrm{O}$ VIII at 0.55 and $0.65 \mathrm{keV}$ may be marginal because of the statistics. The importance of emission lines from the absorbing material is that they emerge from the entire ionized plasma, while absorption features reveal only the properties of material in the line of sight.

As the last example, Fig. 17b shows the expected spectrum from a thermal source of $0.5 \mathrm{keV}$ at the level of $1 \mathrm{mCrab}$. Though the energy resolution at low energy is poorer than at high energy, the sensitivity is high enough that many clear emission lines can be seen with only a $30-\mathrm{ksec}$ observation. If you compare the Astro-E spectrum with that of $A X A F$ shown in the WWW (see Table 1), even for the low-energy spectroscopy below $2 \mathrm{keV}$, the XRS has an advantage over $A X A F$ because of its higher efficiency. Such performance is very useful to examine the hot plasmas surrounding the nuclei of AGN. 

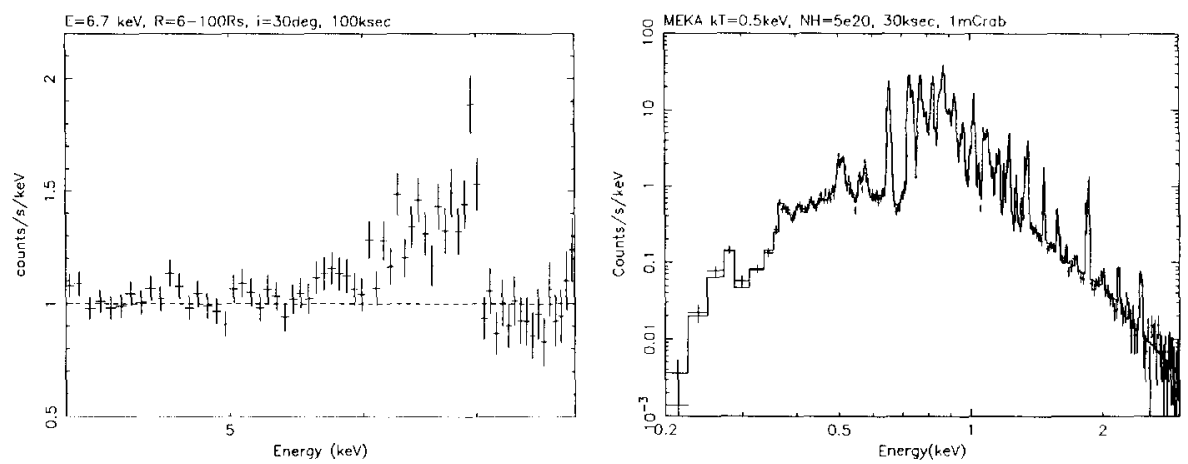

Figure 17. Left: Simulation of disk line $(6.7 \mathrm{keV})$. Right: Simulation of thermal spectrum $(0.5 \mathrm{keV})$.

\subsection{Summary of Future Missions}

The parameters and performance of $A X A F, X M M$, and Astro-E are summarized in Table 1. $A X A F$ is characterized by the high spatial resolution of the telescope. $X M M$ has the largest effective area. Astro- $E$ has the best energy resolution at iron $\mathrm{K}$ line.

In Fig. 18, the effective areas of these missions are presented against X-ray energies, together with $A S C A$ as a reference. It seems to be clear that $A X A F$ will be dedicated for imaging at low energies, while $X M M$ has twice or three times larger area than Astro-E 4 XIS up to several keV. Though Astro-E XRS has only one telescope, it has twice as large an area as one $A S C A$ telescope or $A X A F$ at $7 \mathrm{keV}$.

Figure 19 shows the system effective area of the spectrometers, which is the product of the telescope area and the detector efficiency. Above $2 \mathrm{keV}$, Astro- $E$ XRS has the largest effective area, almost three times larger than $A X A F$ HEG at all energies. Below $2 \mathrm{keV}, X M M$ RGS and Astro-E XRS have comparable area.

The resolving powers $E / d E$ of spectrometers are summarized in Fig. 20. The resolving power gradually increases with energy for non-dispersive spectrometers, such as proportional counters (PC), gas-scintillation proportional counters (GIS), CCDs, and calorimeters (XRS). On the other hand, grating spectrometers have better resolving power at longer wavelengths, because of the diffraction condition. The energy resolution $d E$ at $6-7 \mathrm{keV}$ is shown on the right-hand scale.

If we assume that line broadening is due to the Doppler effect, the $A S C A$ $\mathrm{CCD}$ resolution of $120 \mathrm{eV}$ can reveal the broad iron $\mathrm{K}$ line from a disk rotating at $10,000 \mathrm{~km} \mathrm{~s}^{-1}$ (see the left-hand scale). Astro-E XRS and other spectrometers with $E / d E \geq 600$ can determine velocities higher than $500 \mathrm{~km} \mathrm{~s}^{-1}$, the velocity of NLR clouds. It will also be possible to separate lines from different processes. 
Table 1. Dimensions and Performance of Future X-Ray Missions

\begin{tabular}{|c|c|c|c|}
\hline & $A X A F^{\mathrm{a}}$ & $X M M^{\mathbf{b}}$ & Astro-E \\
\hline Agency & NASA & ESA-NASA & Japan-US \\
\hline Launch Schedule & 1998 & 1999 & Feb 2000 \\
\hline Total Weight (kg) & 4420 & 3900 & 1600 \\
\hline \multicolumn{4}{|l|}{ X-Ray Telescopes } \\
\hline Focal Length (mm) & 10000 & 7500 & $4750 \quad 4500$ \\
\hline Diameter (mm) & 1200 & 350 & 400 \\
\hline Number & 1 & 3 & 4 \\
\hline Coating & Ir & $\mathrm{Au}$ & $\mathrm{Au}$ \\
\hline$S_{\text {eff }}\left(\mathrm{cm}^{-2}\right)$ at $1.5 \mathrm{keV}$ & 1000 & 5000 & $2200 \quad 550$ \\
\hline$S_{\text {eff }}\left(\mathrm{cm}^{-2}\right)$ at $7, \mathrm{keV}$ & 250 & 3000 & $1400 \quad 140$ \\
\hline Angular Resolution & $0^{\prime \prime} .5$ & $20^{\prime \prime}$ & $1^{\prime} .5$ \\
\hline \multirow[t]{2}{*}{$E / d E$ at $6 \mathrm{keV}$} & $50(\mathrm{CCD})$ & $50(\mathrm{CCD})$ & $50(\mathrm{CCD})$ \\
\hline & $200(\mathrm{HEG})$ & $\cdots$ & 500 (Calorimeter) \\
\hline \multirow[t]{3}{*}{$E / d E$ at $1 \mathrm{keV}$} & $20(\mathrm{CCD})$ & $20(\mathrm{CCD})$ & $20(\mathrm{CCD})$ \\
\hline & $1000(\mathrm{HEG})$ & $300(\mathrm{RGS})$ & 200 (Calorimeter) \\
\hline & 300 (LETG) & $\ldots$ & $\ldots$ \\
\hline
\end{tabular}

${ }^{a}$ WWW Homepage: http://hea-www.harvard.edu/asc/axaf-welcome.html

${ }^{b}$ WWW Homepage: http://astro.estec.esa.nl/XMM/xmm.html

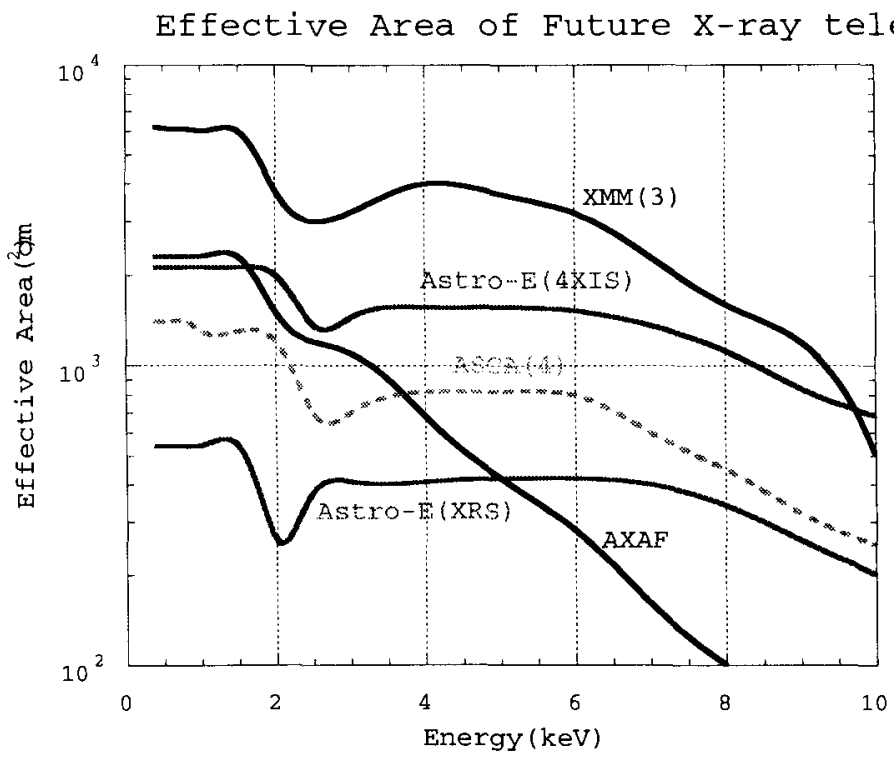

Figure 18. Effective area of future X-ray missions. 


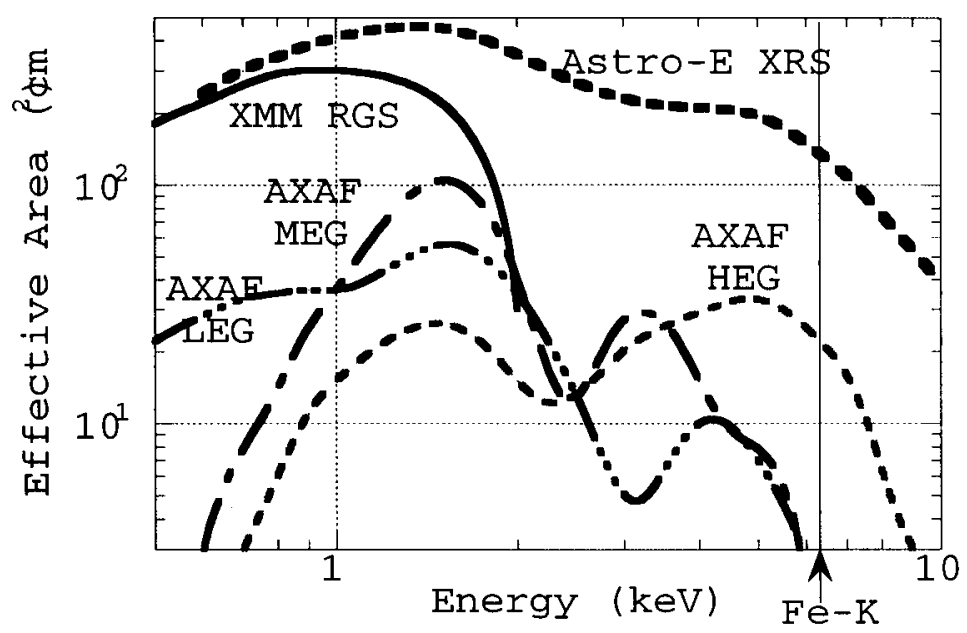

Figure 19. System effective area of future X-ray spectrometers.

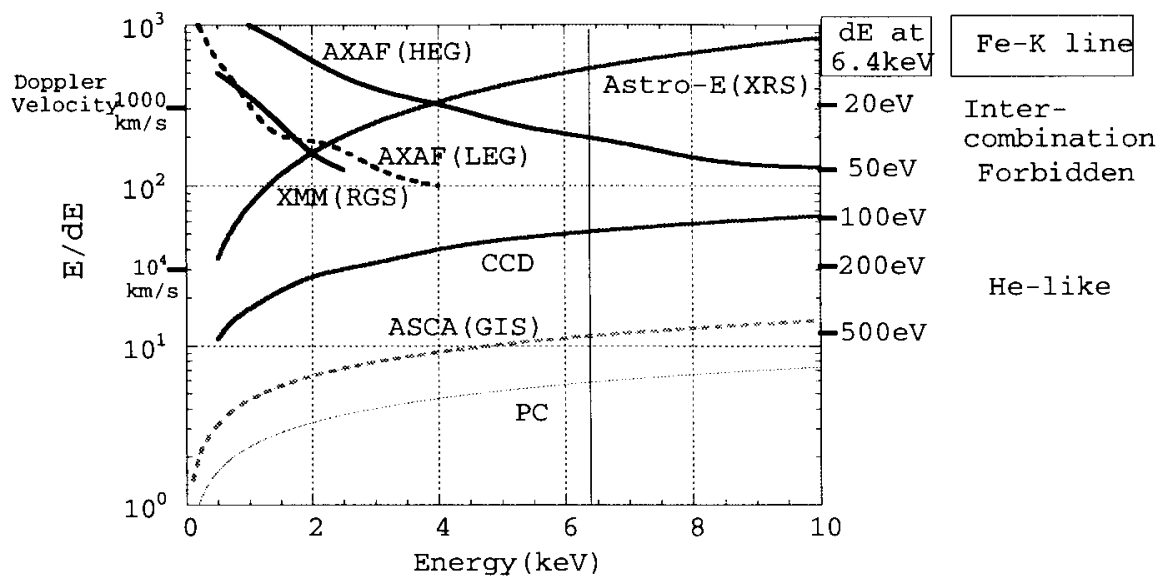

Figure 20. Resolving power of future X-ray spectrometers. 
The PC and GSPC can distinguish the $\mathrm{K}$ emission lines from highly ionized or neutral iron at 6.7 and $6.4 \mathrm{keV}$. If $E / d E$ becomes larger than 100 , forbidden lines may be separated from resonance lines. When $E / d E$ is better than 300 , intercombination lines will be separated.

For the X-ray missions in the 21st century, after these three missions, we are considering the possibility of high-energy telescopes above $20 \mathrm{keV}$. Multilayer coatings are specially designed to enhance reflectivity in a broad energy band by changing the $\mathrm{d}$-spacing from the top to the bottom of layer pairs. A balloon experiment of such a telescope is planned in 1999 to cover the $20-40 \mathrm{keV}$ range and narrow bands peaked at 68 and $78 \mathrm{keV}$ (Serlemitsos et al. 1996). The latter mirrors are to be dedicated to the $\gamma$-ray lines from Ti44 found in young supernova remnants.

\section{Conclusion}

In the next decade, X-ray spectroscopy will achieve for the first time the level of $E / d E \geq 100$, which is common in optical observations. The kinds of science of AGN we will be able to study with such high-energy resolution are as follows:

Iron K-Line Profile: Since the iron lines from AGN are reprocessed by the material around the nucleus, the line width will tell us the orbital velocity $(v>$ $500 \mathrm{~km} \mathrm{~s}^{-1}$ ) of the reprocessor, allowing us to estimate its location. If it is very close to the central black hole, the skewed profile due to the gravitational redshift gives the distance in units of the Schwarzschild radius. If the absolute distance is estimated from X-ray variability time scale, the mass of the black hole will be derived. The line ratio of different ionization states gives the ionization parameter $\Xi=L / n R^{2}$. If we can determine the density $n$ of the material based on the line ratios of the forbidden and resonance lines, we will be able to determine the size or distance $R$.

Low-Energy Lines: Emission lines from hot plasmas can be examined in detail. Line ratios based on the same element in different ionization states can be compared with the electron temperature determined from the continuum spectrum. This will reveal whether the origin of the emission is a photoionized or thermal plasma. It may suggest also that it is not in thermal equilibrium. The line ratios of the different species will allow us to measure the atomic abundance. Such line spectroscopy will provide the best data to confirm or correct atomic data and model calculations currently available.

Finally, it is time for X-ray astronomers to begin spectroscopic studies which will produce real physical parameters: electron temperature $T_{e}$, ionization state, atomic abundance, density $\rho$, size $R$, velocity $v$, and then black-hole masses and so on.

\section{References}

Aschenbach, B. 1987, SPIE, 830, 152.

Awaki, H., et al. 1990, Nature, 346, 544. 
Awaki, H. 1990, Ph.D. Thesis.

Buruke, B., et al. 1991, IEEE Trans. ED-38, 1069.

Canizares, C., \& Kruper, J. 1984, ApJ, 278, L99.

Fabian, A., et al. 1989, MNRAS, 238, 729.

Fabian, A., et al. 1994, PASJ, 46, L59.

Giacconi, R., et al. 1962, Phys. Rev. Lett., 9, 439.

Giacconi, R., et al. 1979, ApJ, 230, 540.

Halpern, J. 1995, Nature, 375, 633.

Iwasawa, K., et al. 1994, PASJ, 46, L167.

Iwasawa, K., et al. 1996, MNRAS, in press.

Kunieda, H. 1995, in 'Highlights of Astronomy', ed. I. Appenzeller, Vol. 10, p. 513.

Makino, F., et al. 1987, Astrophys. Lett. Comm., 25, 223.

Marshall, F., et al. 1992, in 'Frontiers of X-Ray Astronomy', eds. Tanaka \& Koyama, 233.

Mushotzky, R., et al. 1980, ApJ, 235, 377.

Nandra, K., \& Pounds, K. 1994, MNRAS, 268, 405.

Nandra, K., \& Pounds, K. 1992, Nature, 359, 215.

Nandra, K., et al. 1996, ApJ, in press.

Ohashi, T., et al. 1996, PASJ, 48, 157.

Ohtani, C., et al. 1996, PASJ, 48, 211.

Petre, R., \& Serlemitsos, P. 1985, Appl. Opt., 24, 1833.

Pfeffermann, E., et al. 1986, SPIE, 733, 519.

Rothschild, R., et al. 1979, Space Sci. Inst., 4, 265.

Serlemitsos, P. 1988, Appl. Opt., 27, 1447.

Serlemitsos, P., et al. 1995, PASJ, 47, 105.

Serlemitsos, P., et al. 1996, in 'X-Ray Imaging and Spectroscopy of Cosmic Hot Plasmas', in press.

Tanaka, K. 1986, PASJ, 38, 225.

Tanaka, Y., et al. 1994, PASJ, 46, L37.

Tanaka, Y., et al. 1995, Nature, 375, 659.

Tennant, A., \& Mushotzky, R. 1983, ApJ, 264, 92.

Tsuru, T., et al. 1994, in 'New Horizons of X-Ray Astronomy', eds. Makino \& Ohashi, p. 529.

Turner, M., et al. 1989, PASJ, 41, 345.

Wood, K. 1984, ApJS, 56, 507. 